\title{
MANAGEMENT OF BIG DATA IN CONTEMPORARY WORLD
}

\author{
Anjaneyulu, J. \\ Doctoral Candidate, Universitat Oberta De Catalunya, Spain \\ e-mail: ajinugu@gmail.com \\ Madhavi Lalitha, V V. \\ Doctoral Candidate, IIHMR University, Jaipur, India \\ e-mail: jinuguamdhavi@gamil.com \\ Sreechandana, $\mathbf{K}$. \\ B.Tech. (Bio-medical Engg.), Osmania University, Hyderabad, India. \\ e-mail: sreechandanakodimela@gmail.com
}

\begin{abstract}
:
As it is strongly believed that technology can reap its best only when it can be tamed by all stakeholders. Big data technology has no exception for this and even after a decade of emergence, the technology is still a herculean task and is in nascent stage with respect to applicability for many people. Having understood the gaps in the technology adoption for big data in the contemporary world, the present exploratory research work intended to highlight the possible prospects of big data technologies. It is also advocated as to how the challenges of various fields can be converted as opportunities with the shift in the perspective towards this evolving concept. Examples of apex organizations like (IMF and ITU) and their initiatives of big data technolgies with respect to the Sustainable Development Goals (SDGs) are also cited for a broader outlook. The intervention of the responsible organizations along with the respective governments is also much sought for encouraging the technology adoption across all the sections of the market players.
\end{abstract}

\section{Keywords:}

Internet of Things (IOT), Sustainable Development Goals (SDG), Big Data, Internet Data Sources (IDS), Challenges

\section{Introduction:}

Big data, being an emerging concept of the contemporary world, has become very popular far and wide. The applications of the concept also made it all pervasive across various sectors like Internet of Things (IOT), healthcare, social networks, scientific projects and much evolving smart technologies as well as their utilities. Be it the nowcasting method or forecasting method, harnessing the big data has become possible with such various disruptive technologies/techniques/methodologies (Bok et al., 2017). Because of the popularity that the big data management has gained, the concept has spread from $3 \mathrm{Vs}$ (Volume, Velocity and Variety) to 5Vs (Volume, Velocity, Variety, Veracity and Value) and then to 10Vs (Volume, Variety, Validity, Variability, Vocabulary, Vagueness, Veracity, Velocity, Venue and Value) (Tiwari et al., 2018; Assunção et al., 2015; Robak et al., 2014). as shown in the following figure -1 . 


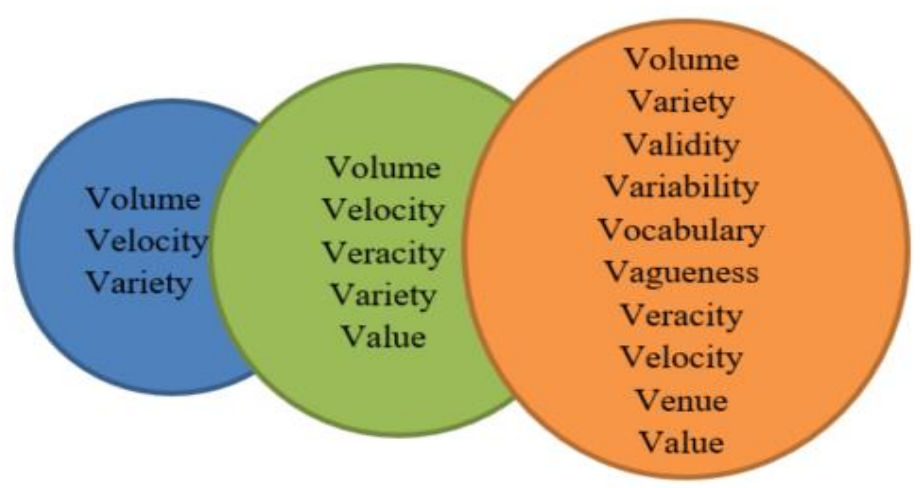

Figure - 1: Evolution of the Vs of Big Data

However, many problems still persist in dealing with the big data and for many people big data technology is still an alien language not only to understand but also to implement (Robak et al., 2014). These problems are encountered across various stages like data generation, storage, processing and analysing and hence it has become inevitable to the organizations to update their technologies or IT infrastructure from time to time, which is always a risk (Vozábal, 2016).

It is no doubt that the contemporary world is zooming day-by-day towards excellence. But many small and medium players of the world are falling behind the run of excellence (. Of late, in the wake of Sustainable Development Goals (SDGs), even the governments are exploring the big data methodologies to reach their respective goals and are compelling their stakeholders to adopt the same (Klein and Verhulst, 2017). In addition to the challenges with respect to the set up or update of technology as well as methodological problems in the process of generating and analysing data have increased the challenges by manifold (Zhang et al., 2018). For example, the risks that arise in the process of data organization (like representativeness of the sample) from various Internet Data Sources (IDS) coupled with domain (such as healthcare, agriculture, education etc.,) specific issues underpin the necessity to hone the technology in an optimized manner (Beresewicz, 2017).

Having understood the scenario of big data and the allied concepts and the risks involved, the present paper tries to highlight the challenges along with advocating the possible prospects. The growing impetus of the big data technologies vis-à-vis the challenges in the contemporary world that are not addressed is the rationale of the study. This exploratory study gathers the information regarding the above discussed topic in a systematic manner by dealing with evolution, management and the prospects of the big data technologies by underpinning the problems encountered throughout the process continuum. Possible solutions for the domain specific challenges are advocated, limitations are discussed, and future research prospects are also highlighted for research aspirants. 


\section{Review of Literature:}

Change is constant in the contemporary world which is characterized by technological innovation coupled with optimum utilization. So, the change of paradigm in the Official Statistics has proposed to consider the existing data rather generating new data for drawing inferences (Beresewicz, 2017). This concept has led to the potential source of statistics, which is big data. Having introduced in 1997 by Cox and Ellsworth in an Association for Computing Machinery (ACM) article, big data concept has got the ability to answer the burgeoning questions raised across the globe for researchers, policy makers and businesses (Tiwari et al., 2018; ).

\section{Definitions of Big Data}

As big data concept is applicable to numerous fields, its definitions are also numerous which are stated as per the perception of the user. Though there is no common consensus on the definition of big data, few considered definitions are as follows.

A possible definition of big data which includes all the characteristics (unstructured, voluminous, variety etc.,) is the big data are highly detailed exhaust data automatically captured by sensors or generated during the use of IT systems

- Beręsewicz et al., (2018), EU.

The three characteristics of big data known as the $3 \mathrm{~V}$ 's - (high) volume, (high) velocity and (high) variety

- Laney (2001)

A new generation of technologies that used to economically extract value from very large volumes of a wide variety of data by enabling high-velocity capture, discovery, and/or analysis.

$-I D C, U S$

Big data is not a single product rather a complex set of technologies and techniques for data gathering, data store, data processing, data access, data analytics and data visualisation.

- Sheikh (2013)

Thus, there are many versions of the definition and researchers perceived big data concept with respective domain specific expertise. The allied terms like big data, big data analytics and related are coined in many robust and matured fields like Information Technology, Education, Oceanography, Banking, Transport and others (Young, 2007).

\section{Management of Big Data}

As the global players are heading towards the voluminous data from various sources (big data), it is quite important to manage the data effectively from time to time (Brahmia et al., 2019). Discussing the significance of big data analytics in healthcare field, De Silva et al. (2015) highlighted that due to the mismatch between the electronic health records data and the format required for big data analytics, the management of healthcare data is not effective. So, organization of the data is a major requirement for seamless analysis of the 
data and hence the inference. The competitiveness created due to big data management is benefiting data intensive organizations only and the less-technology intense organization are lagging behind in the race (Brock and Khan, 2017).

Assunção et al. (2015) cited the open challenges that are encountered in the processes of administering the big data, because of which many players have been still using legacy systems. They are., data variety, data integration, data processing and resource management. Similarly, the role of governance in managing big data is stressed by UN report (2014). It is mentioned in the report that as many stakeholders are involved in the process of big data management, like public, private institutions, civil society as well as academia, it is the duty of the government to safeguard the interest of public and ensure safety of the public data. This can only be possible through tightening the governance and legal systems with apt models and frameworks.

\section{Prospects of Big Data}

The growing characteristics of big data not only pose challenges to the intellectual fraternity but also provide means for umpteen prospects in plethora of fields (social sciences, economics, healthcare, engineering and others) (Hammer et al., 2017). The dimensions of Vs that emerged over time has given in the following figure-2 and for along each dimension, prospects can be planned.

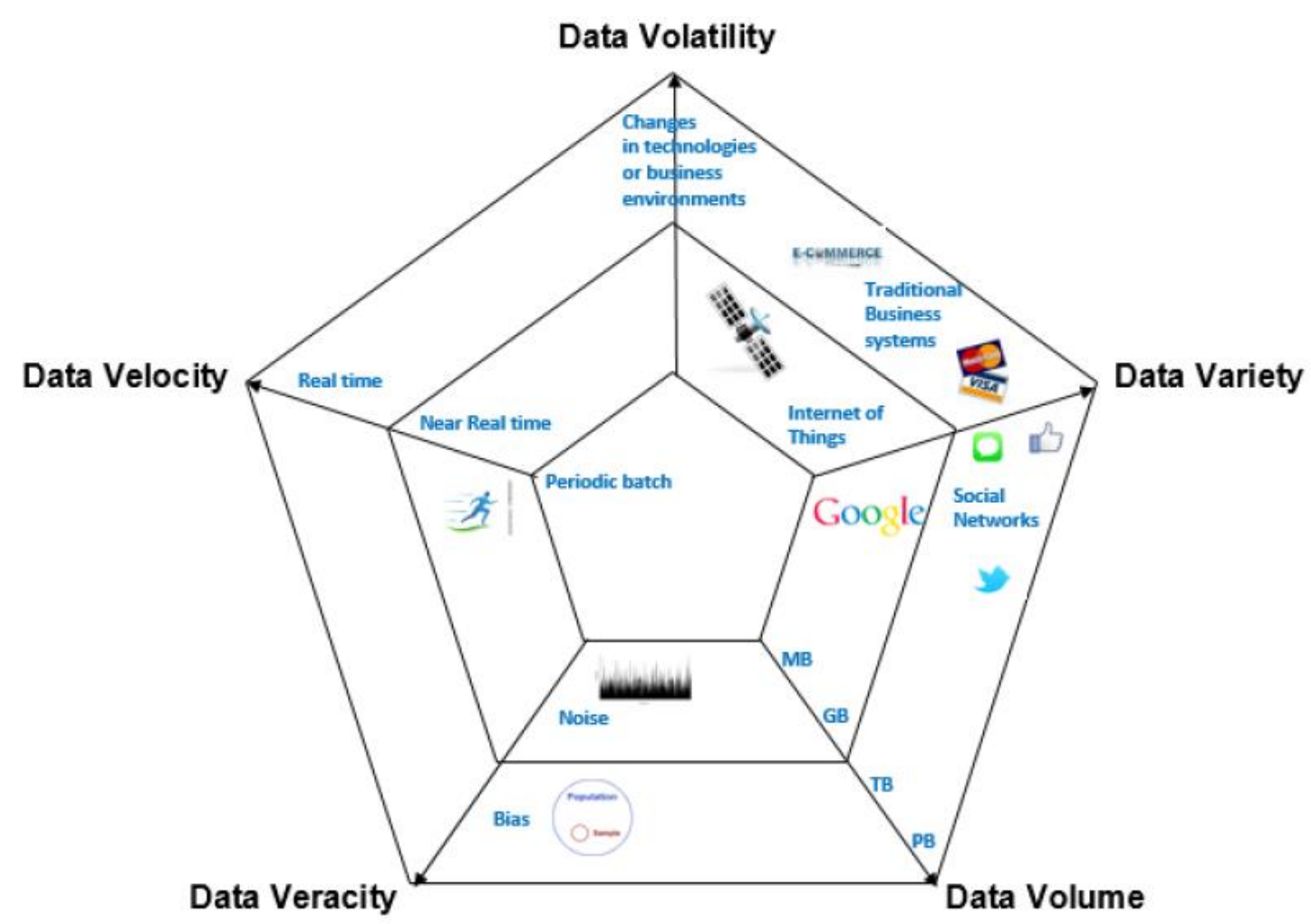

Figure -2: Dimensions of Vs and the corresponding changes (Source: Hammer et al., 2017) 
Likewise, the prospects of the concept of big data have to be emerged by keeping the challenges in view and also the growing global requirements. This is very essential because, ubiquitously there are growing requirements with respect to theoretical and technological evolution as well as applications of big data (Farhaoui and Moussaid, 2019). For example, the enormous data that is generated directly or indirectly has given impetus to Data Driven Innovation (DDI), which in turn influenced the ICT and non-ICT (healthcare, transport, retail sectors and others) (OECD, 2015). So, needless to say that there are emerging prospects of big data in all the data driven areas. However, in all the above cited sectors there are always challenges with respects to the Vs stated (3Vs or $5 \mathrm{Vs}$ or $10 \mathrm{Vs}$ ). Tapping the challenges and obviously provide prospects in the respective areas (Assunção et al., 2015).

Hamid Ekbia et al. (2015) advocated another paradigm of looking for prospects of big data with respect to various perspectives, viz., Product-Oriented Perspective: The Scale of Change; Process-Oriented Perspective: Pushing the Technological Frontier; CognitionOriented Perspective: Whither the Human Mind; and The Social Movement Perspective: The Gap Between Vision and Reality. That is, new dimensions, the dilemmas and the extent of applications that arise by considering various perspectives can unleash many prospects of big data technologies. Explaining how technology can be harnessed to realize the 17 SDGs, the report of International Telecommunication Union (ITU) listed all the possible prospects of big data in order to overcome the challenges as well as to reach the laid goals quickly.

\section{Conclusions:}

The much-discussed concept, big data is being defined in various ways and is adopted to the processes across the globe. But the problems that are encountered in the run to be on par with the burgeoning technology by the contemporary players of medium and small industries are not much highlighted in the research works. It is pointed by the intellectual community that the technology has to be harnessed so that the means and the fruits can be utilized by all the sections of the stakeholders. With this aim, the present study tried to highlight the possible prospects that the big data concept/technology has across various domains. Also, the need for the shift in the thought process to look at several perspectives is also discussed. The concept is bolstered with the examples with respect to the initiatives of SDGs that are advocated by international organizations.

Though the possible prospects are discussed for much fervour, adaptability of the small and medium players depends on sheer applicability and ease of taming the technology. This gap in theory and application is omnipotent with respect to any technology. Hence, all the stakeholders of intellectual fraternity (software developers, hardware techies, data scientists, researchers, end users and others) have to identity the weakest link in the big data technology adoption chain in the contemporary world and have to strengthen it with apt methodologies and techniques. Failing which, expecting the technology adoption by all the players of contemporary world would be like mopping the floor by keeping the tap open. 


\section{Recommendations:}

As any shift in the society is not completely possible without the active intervention of policies and procedures, it is the role of the governments to encourage and develop systems that support the applications of big data technologies. This approach is much needed for the contemporary society which is mostly characterised by disruptive innovations and is the dire necessity for the developing countries. With the initiation of the regulatory authorities, the sense of data security and trust will be fostered among all the sections of the society and hence the small and medium players can also pick up with the race of big data technologies along with their contemporaries.

\section{References:}

1. Bok, B., Caratelli, D., Giannone, D., Sbordone, A. and Tambalotti, A. (2017), "Macroeconomic Nowcasting and Forecasting with Big Data", Federal Reserve Bank of New York Staff Reports Report No. 830, November 2017.

2. Tiwari, S., Wee, H.M. and Daryanto, Y. (2018), "Big data analytics in supply chain management between 2010 and 2016: Insights to industries", Computers \& Industrial Engineering, Vol. 115, pp. 319-330.

3. Robak, S., Franczyk, B., Robak, M. (2014). Research problems associated with big data utilization in logistics and supply chains design and management. 2014 Federated Conference on Computer Science and Information System.

4. Vozábal, M. (2016), "Tools and Methods for Big Data Analysis, Master Thesis" University of West Bohemia Faculty of Applied Sciences, Department of Computer Science and Engineering, Pilsen.

5. Klein, T. and S. Verhulst (2017), "Access to new data sources for statistics: Business models and incentives for the corporate sector", OECD Statistics Working Papers, 2017/06, OECD Publishing, Paris. http://dx.doi.org/10.1787/9a1fa77f-en

6. Zhang, Z., He, Q., Gao, J. and Ni, Ming. (2018), “A deep learning approach for detecting traffic accidents from social media data", Transportation Research Part C, Vol. 86, pp. 580-596.

7. Robak, S., Franczyk, B. and Robak, M. (2014), "Research Problems Associated with Big Data Utilization in Logistics and Supply Chains Design and Management", Position papers of the 2014 Federated Conference on Computer Science and Information Systems Vol. 3, pp. 245-249, DOI: 10.15439/2014F472, ACSIS.

8. Beresewicz, M. (2017), “A Two-Step Procedure to Measure Representativeness of Internet Data Sources", International Statistical Review, Vol. 85 No. 3, pp. 473493. doi:10.1111/insr.12217 
9. UN Report (2014), "A World that Counts Mobilising the Data Revolution for Sustainable Development, by the Independent Expert Advisory Group on a Data Revolution for Sustainable Development, November.

10. De Silva, D., Burstein, F., Jelinek, H. and Stranieri, A. (2015), "Addressing the Complexities of Big Data Analytics in Healthcare: The Diabetes Screening Case", Australasian Journal of Information Systems, Vol. 19, pp. S99-S115.

11. Beręsewicz, M., Lehtonen, R., Reis, F., Di Consiglio, L. And Karlberg, M. (2018), "An Overview Of Methods For Treating Selectivity In Big Data Sources", European Union.

12. Laney, D. (2001), “3D Data Management: Controlling data volume, velocity and variety”, META Group.

13. Young, J. M. (2007), "An Epidemiology Of Big Data", Doctoral Thesis, Georgetown University of Maryland, USA

14. Brahmia, S., Brahmia, Z., Grandi, F. and Bouaziz, R. (2019), "Managing Temporal and Versioning Aspects of JSON-Based Big Data via the JSchema Framework", ICBDSDE 2018, SBD 53, pp. 27-39, 2019

15. Brock, V. F. and Khan, H. U. (2017), “Are enterprises ready for big data analytics? A survey-based approach, Int. J. Business Information Systems, Vol. 25 No. 2, pp. 256-277.

16. Assunção, M. D., Calheiros R. N., Bianchi, S., Netto, M.A.S. and Buyya., R. (2015), "Big Data computing and clouds: Trends and future directions", J. Parallel Distrib. Comput. Vol. 79-80, pp. 3-15.

17. Sheikh, N. (2013) 'Chapter 11 - big data, hadoop, and cloud computing', in Sheikh, N. (Ed.): Implementing Analytics, Morgan Kaufmann, Boston.

18. Farhaoui, Y. and Moussaid, L. (2019), "Big Data and Smart Digital Environment", Studies in Big Data 53, https://doi.org/10.1007/978-3-030-12048-1.

19. Hammer, C. L., Diane C. Kostroch, D. C., Quirós, G. and STA Internal Group (2017), "Big Data: Potential, Challenges, and Statistical Implications", IMF Staff Discussion Note, September 2017, SDN/17/06.

20. OECD (2015), Data-Driven Innovation: Big Data for Growth and Well-Being, OECD Publishing, Paris. http://dx.doi.org/10.1787/9789264229358-en

21. Hamid Ekbia et al. (2015), “Advances In Information Science Big Data, Bigger Dilemmas: A Critical Review", Journal of The Association For Information Science And Technology, 66(8):1523-1545, 2015 
22. ITU (2017), "Fast-forward progress Leveraging tech to achieve the global goals", https://www.itu.int/pub/S-GEN-ICTS.01 